\title{
Surface photometry of WINGS galaxies with GASPHOT`
}

\author{
M. D’Onofrio ${ }^{1}$, D. Bindoni ${ }^{1}$, G. Fasano ${ }^{2}$, D. Bettoni ${ }^{2}$, A. Cava ${ }^{3}$, J. Fritz ${ }^{4}$, M. Gullieuszik ${ }^{2}$, \\ P. Kjærgaard ${ }^{5}$, A. Moretti ${ }^{1}$, M. Moles ${ }^{6}$, A. Omizzolo ${ }^{7,2}$, B. M. Poggianti ${ }^{2}$, T. Valentinuzzi ${ }^{1}$, and J. Varela ${ }^{6}$ \\ 1 Department of Physics and Astronomy, University of Padova, Vicolo Osservatorio 3, 35122 Padova, Italy \\ e-mail: mauro.donofrio@unipd.it \\ 2 INAF - Padova Astronomical Observatory, Vicolo Osservatorio 5, 35122 Padova, Italy \\ 3 Observatoire de Genève, Université de Genève, $51 \mathrm{Ch}$. des Maillettes, 1290 Versoix, Switzerland \\ ${ }^{4}$ Sterrenkundig Observatorium, University of Gent Krijgslaan 281 S9, 9000 Gent, Belgium \\ 5 The Niels Bohr Institute for Astronomy Physics and Geophysics, J, Maries Vej 30, 2100 Copenhagen, Denmark \\ ${ }^{6}$ Centro de Estudios de Física del Cosmo de Aragon, Plaza San Juan 1, 44001 Teruel, Spain \\ 7 Specola Vaticana, 00120 Stato Città del Vaticano, Italy
}

Received 30 May 2014 / Accepted 8 October 2014

\begin{abstract}
Aims. We present the $B, V$, and $K$ band surface photometry catalogs obtained by running the automatic software GASPHOT on galaxies from the WINGS cluster survey with isophotal areas larger than 200 pixels. The catalogs can be downloaded at the Centre de Données Astronomiques de Strasbourg.

Methods. The luminosity growth curves of stars and galaxies in a given catalog relative to a given cluster image were obtained simultaneously by slicing the image with a fixed surface brightness step in several SExtractor runs. Then, using a single Sersic law convolved with a space-varying point spread function (PSF), GASPHOT performed a simultaneous $\chi^{2}$ best-fit of the major- and minor-axis luminosity growth curves of galaxies. We outline the GASPHOT performances and compare our surface photometry with that obtained by SExtractor, GALFIT, and GIM2D. This analysis is aimed at providing statistical information about the accuracy that is generally achieved by the softwares for automatic surface photometry of galaxies.

Results. The GASPHOT catalogs provide the parameters of the Sersic law that fit the luminosity profiles for each galaxy and for each photometric band. They are the sky coordinates of the galaxy center (RA, Dec), the total magnitude $(m)$, the semi-major axis of the effective isophote $\left(R_{\mathrm{e}}\right)$, the Sersic index $(n)$, the axis ratio $(b / a)$, and a flag parameter $\left(Q_{\mathrm{FLAG}}\right)$ that generally indicates the fit quality. The WINGS-GASPHOT database includes 41463 galaxies in the $B$ band, 42275 in the $V$ band, and 71687 in the $K$ band. The bright early-type galaxies have higher Sersic indices and larger effective radii, as well as redder colors in their center. In general, the effective radii increase systematically from the $K$ to the $V$ and $B$ band.

Conclusions. The GASPHOT photometry agrees well with the surface photometry obtained by GALFIT and GIM2D, and with the aperture photometry provided by SExtractor. In particular, the direct comparison of structural parameters derived by different softwares for common galaxies indicates that the systematic differences are small in general. The only significant deviations are most likely due to the peculiar (and very accurate) image processing adopted by WINGS for large galaxies. The main advantages of GASPHOT with respect to other tools are (i) the automatic finding of the local PSF; (ii) the short CPU execution time; and (iii) the remarkable stability against the choice of the initial-guess parameters. All these characteristics make GASPHOT an ideal tool for blind surface photometry of large galaxy samples in wide-field CCD mosaics.
\end{abstract}

Key words. catalogs - galaxies: clusters: general - surveys

\section{Introduction}

Thanks to the performances of modern CCD detectors and computing systems, several astronomical surveys have had the opportunity of mapping large sky areas, thus in turn providing the opportunity of measuring photometric and structural properties of thousands of extended sources using relatively short exposure-time imaging. This has been achieved also thanks to automatic surface photometry tools that offer reliable results and are not heavily demanding in terms of computer time. The tools most widely used for the aperture and surface photometry of galaxies are SExtractor (Bertin \& Arnouts 1996), GIM2D (Simard 1998), and GALFIT (Peng et al. 2002).

* Catalogs are only available at the CDS via anonymous ftp to cdsarc.u-strasbg.fr (130.79.128.5) or via http://cdsarc.u-strasbg.fr/viz-bin/qcat?]/A+A/572/A87
In the framework of the Wide-field Imaging of Nearby Galaxy-clusters Survey ${ }^{1}$ (WINGS; Fasano et al. 2006), we have devised the tool GAlaxy Surface PHOTometry (GASPHOT; Pignatelli et al. 2006), aimed at performing the automatic surface photometry of large galaxy samples. The performances of GASPHOT have been already tested on simulated galaxies and against the results of supervised, single-object photometry by Pignatelli et al. (2006).

In the present paper we present the catalogs obtained by running GASPHOT on the $B, V$, and $K$ band wide-field imaging of the WINGS survey. Moreover, we compare the results of GASPHOT with those obtained by SExtractor, GIM2D, and GALFIT. In particular, the comparison with GIM2D was made using the catalogs obtained runnig GASPHOT on the

\footnotetext{
1 See the WINGS web-site for all the details of this project at http://web.oapd.inaf.it/wings
} 
$B$ band imaging of the Millennium Galaxy Catalog (MGC) survey (Allen et al. 2006) and partly published in Poggianti et al. (2013a).

The paper is organized as follows: in Sect. 2 we recall the features of the WINGS survey to set the context of the GASPHOT database. Section 3 describes the guidelines of the software, the data sample analyzed by GASPHOT, and the typical output files. In Sect. 4 the GASPHOT photometry is compared with that from SExtractor, GIM2D, and GALFIT. In Sect. 5, using the structural parameters from GASPHOT, we present the main scaling relations of the WINGS galaxies in the different photometric bands. Our conclusions are drawn in Sect. 6.

\section{The WINGS survey}

The WINGS survey (Fasano et al. 2006) is a long-term project dedicated to providing a reliable characterization of the photometric and spectroscopic properties of galaxies in nearby clusters. The core of the survey is WINGS-OPT (Varela et al. 2009), which is a set of $B$ and $V$ band images of a complete, X-ray selected sample of 77 clusters with redshift $0.04<z<0.07$. The images have been taken with the Wide Field Camera (WFC, $34^{\prime} \times 34^{\prime}$ ) at the INT-2.5 $\mathrm{m}$ telescope in La Palma (Canary Islands, Spain) and with the Wide Field Imager (WFI, $34^{\prime} \times 33^{\prime}$ ) at the MPG/ESO $2.2 \mathrm{~m}$ telescope in La Silla (Chile). The optical photometric catalogs have been obtained using SExtractor and are $90 \%$ complete at $V \sim 21.7$, which translates into $M_{V}^{*}+6$ at the mean redshift of the survey (Varela et al. 2009). The WINGS-OPT catalogs contain $\sim 400000$ galaxies in both the $V$ - and $B$-band. According to Varela et al. (2009, Table D.2), in the whole cluster sample the surface brightness limits at $1.5 \sigma_{\mathrm{bkg}}$ ( $\sigma_{\mathrm{bkg}}$ is the standard deviation per pixel of the background) span the ranges 24.7-26.1 (average value: 25.71) in the $V$ band and 25.4-26.9 (average value: 26.39) in the $B$ band.

SExtractor catalogs have also been obtained for the nearinfrared follow-up of the survey (WINGS-NIR; Valentinuzzi et al. 2009), which consists of $J$ - and $K$-band imaging of a subsample of 28 clusters of the WINGS-OPT sample, taken with the WFCAM camera at the UKIRT telescope. Each mosaic image covers $\approx 0.79 \mathrm{deg}^{2}$. With the SExtractor analysis the $90 \%$ detection rate limit for galaxies is reached at $J=20.5$ and $K=19.4$. The WINGS-NIR catalogs contain $\sim 490000$ in the $K$ band and $\sim 260000$ galaxies in the $J$ band. The photometric depth of the WINGS-NIR imaging is slightly worse than that of the WINGS-OPT imaging. Thus, for the $K$-band the surface brightness limit at $1.5 \sigma_{\mathrm{bkg}}$ spans the range $20.6-21.5$ (see Table 4 in Valentinuzzi et al. 2009, average value: 21.15 ).

To give a more complete sketch of the observing material available for WINGS, we just mention that the survey also includes medium-resolution, multifiber spectroscopy (WINGS-SPE) and $U$-band photometry (WINGS-UV) of galaxies in subsamples of the WINGS-OPT cluster sample. We refer to Cava et al. (2009) and Omizzolo et al. (2014) for details about these follow-ups. Finally, we gather $B, V$ and $u^{\prime}$ band imaging with OmegaCam at VST (one square degree FOV) of the WINGS clusters in the southern hemisphere (Gullieuszik et al., in prep.).

In addition to the aperture photometry catalogs (SExtractor) and the surface photometry catalogs presented here, this huge amount of data has produced morphological catalogs ( 40 000 galaxies) obtained using the dedicated automatic tool MORPHOT (Fasano et al. 2012) and spectroscopic catalogs including redshifts (Cava et al. 2009), star formation histories, stellar masses and ages (Fritz et al. 2011), as well as equivalent widths and line-indices (Fritz et al. 2014) of $~ 6000$ galaxies.

A complete description of the WINGS database, including the GASPHOT catalogs presented here, can be found in Moretti et al. (2014).

\section{Galaxy sample and the GASPHOT catalogs}

The surface photometry of galaxies in the WINGS clusters was performed on the same sample for the morphological (MORPHOT) analysis, that is $\sim 40000$ galaxies with an isophotal area larger than 200 pixels at the threshold of $2.5 \sigma_{\text {bkg }}$. To handle such a large number of galaxies, the automatic tool GASPHOT was devised. Details about the software are given in Pignatelli et al. (2006), together with tests of the GASPHOT performances, mainly based on simulated galaxy samples. The code first produces a set of growth curves of stars and galaxies through several runs of SExtractor. Then, a simultaneous best-fit of the major- and minor-axes growth curves is performed for each galaxy using a single 2D Sersic law convolved with a space-varying point spread funtion (PSF). The fitting strategy of GASPHOT is a hybrid between the $1 \mathrm{D}$ equivalent luminosity profile fitting and the $2 \mathrm{D}$ full-image fitting technique. Advantages and disadvantages of these approaches are outlined in Sect. 4.2.6.

The GASPHOT tool is blind, meaning that it performs the surface photometry of all galaxies in a given catalog (relative to a given frame) without requiring a first guess of the model parameters for each galaxy, as is the case for most popular 2D tools. GASPHOT only needs a careful choice of the configuration file parameters that influence the observed (PSF convolved) light profiles, in particular the deblending parameter and the detection and analysis threshold parameters of SExtractor (DEBLEND_NTHRESH, DETEC_THRESH, and ANALYSIS_THRESH), the surface brightness step, and the magnitude range of the stars used to derive the PSF profile (Pignatelli et al. 2006).

After extracting the major- and minor- axis growth curves, the best-fit procedure provides for each galaxy the total magnitude $(m)$, the axis ratio $(b / a)$, the effective radius $\left(R_{\mathrm{e}}\right)$, the Sersic index $(n)$, and the $\chi^{2}$ of the best-fit Sersic model. We recall that, since the boundary values used by GASPHOT for $n$ are 0.5 and 8 , finding these output values of the Sersic index is considered as an indication that the best-fit procedure has been problematic or unsuccessful.

Although GASPHOT considers the background as a free parameter of the best-fit algorithm, it is convenient in most cases to operate with images in which the background has already been roughly subtracted (for instance, with SExtractor) and to refine the subtraction that limits the range of variability of the background parameter in the fitting procedure. Varela et al. (2009) described the procedure of background subtraction adopted for the WINGS clusters in detail. Here we only recall that the careful modeling and removal of the brightest galaxies and stars (most of them equipped with extended halos) allows obtaining reliable surface photometry parameters of both the bright galaxies themselves and the many small or faint companion galaxies that are embedded inside their halos. This procedure obviously also results in a very precise determination of the background path to be subtracted from the images. Thus, we allowed GASPHOT to vary the background parameter by only $1.8 \times \sigma_{\mathrm{bkg}}$.

The CPU time needed to run GASPHOT on a sample of $\sim 600$ galaxies (the typical number of galaxies in WINGS cluster 
catalogs) is $\sim 2 \mathrm{~h}$ on a server with a double CPU Xeon E5439 at $2.6 \mathrm{GHz}$ (eight cores in total) with 16Gb RAM. Most of this time is used by SExtractor to extract the major- and minoraxis growth curves.

Pignatelli et al. (2006) compared the output parameters of GASPHOT with results of GIM2D and GALFIT using $\sim 15000$ simulated galaxies, including multicomponent $\left(r^{1 / 4}+\right.$ exp.) galaxies and blended objects, in a wide range of magnitude, flattening, and radius. They found that for objects with a threshold isophotal area greater than 200 pixels, the photometric and structural parameters derived by GASPHOT agree very well with the simulation input values, even for composite luminosity profiles, blended objects, and low surface brightness galaxies. A small number of outliers were found, but the results were robust in a statistical sense. The scatter was small in general $(<15 \%)$, but for single objects the errors on effective radius $R_{\mathrm{e}}$ and Sersic index $n$ could (in a few cases) exceed 20-40\%. Finally, although giving similar results on simulated galaxies, GALFIT and GIM2D were found to be less robust than GASPHOT when using a single Sersic model to fit luminosity profiles of real galaxies. The last result relied on a small sample of galaxies with detailed (visually supervised) surface photometry.

The GASPHOT WINGS-OPT sample in the $B(V)$ band consists of 41463 (42275) galaxies of all morphological types detected by SExtractor as having an isophotal area larger than 200 pixels above $2.5 \sigma_{\mathrm{bkg}}$. The average number of galaxies per cluster that satisfy this condition is $\sim 560$. Using the same criterion, the GASPHOT WINGS-NIR sample consists of 71687 galaxies in the subsample of 28 clusters observed in the $K$ band (Valentinuzzi et al. 2009; 2750 objects per cluster, on average). The galaxies in common among the $B, V$ and $K$ bands are $\sim 10424$ and belong to 25 clusters $^{2}$.

For each galaxy, the GASPHOT catalogs list the WINGS identifier and the best-fit parameters found by GASPHOT for the single Sersic law model, in particular, the coordinates (RA and Dec) of the center, the total magnitude, the effective radius, the Sersic index and the axis ratio (see Table 1). Moreover, for each galaxy, GASPHOT provides the major- and minor-axis grow curves, and the ellipticity and position angle profiles of the ellipses that best-fit the isophotes. These can be useful to analyze the shape of galaxies, in particular to test whether there are bars.

The quality of the GASPHOT fit cannot always be judged on the basis of the $\chi^{2}$ parameter because several effects might influence its value. The uncertainty on the background value (in particular close to very bright objects), the choice of the deblending parameters of SExtractor for luminosity profile extraction, the cutting of luminosity profiles for objects close to the CCD borders, the accuracy of the local PSF and, most of all, galaxy substructures that cannot be represented by the Sersic law, especially when objects are well resolved. On the other hand, the errors provided by GASPHOT for each output parameter are usually too small, because they are only formal uncertainties associated with the fitting procedure. For these reasons, neither the $\chi^{2}$ nor the errors on individual parameters have been included in the catalogs. Instead, we preferred to provide possible users with the global quality index $Q_{\mathrm{FLAG}}$. This is a decimal number corresponding to a binary eight-digit number. The first two digits are always set to 0 , while the remaining six are set to 1 when the Sersic index is equal to 0.5 or 8 (third digit), the errors

2 A119, A500, A602, A957x, A1069, A1291, A1631a, A1644, A1795, A1831, A1983, A2107, A2124, A2149, A2169, A2382, A2399, A2457, A2572a, A2589, IIZW108, MKW3s, RX1022, RX1740, Z8338.
Table 1. Parameters provided by GASPHOT for each galaxy in the $V$ band.

\begin{tabular}{lll}
\hline \hline Parameter & Units & Description \\
\hline ID_WINGS & NULL & WINGS object identification \\
RA & {$[\mathrm{deg}]$} & Central right ascension \\
DEC & {$[\mathrm{deg}]$} & Central declination \\
$m_{V}$ & {$[\mathrm{mag}]$} & Total magnitude \\
$R_{\mathrm{e}}$ & [arcsec] & Major-axis effective radius \\
$n$ & NULL & Sersic index \\
$b / a$ & NULL & Axis ratio \\
$Q_{\text {FLAG }}$ & NULL & Quality FLAG \\
\hline
\end{tabular}

in the estimated parameters (magnitude:fourth digit; effective radius:fifth digit; Sersic index:sixth digit; background:seventh digit and axial ratio:eighth digit) exceed the 98 percentile of the error distributions for the given image. For instance, $Q_{\text {FLAG }}=0$ for good fits, 32 for fits that have a Sersic index equal to 0.5 or 8 (the search interval boundaries used by GASPHOT for the Sersic index), 2 for fits with a too large error on the background estimation, 16 for fits with a too large error on the estimated magnitude.

The $Q_{\text {FLAG }}$ parameter is only an attempt to quantify the problems encountered during the fit of each galaxy. A reliable estimate of the uncertainties of GASPHOT can be obtained only in a statistical sense. The comparison of GASPHOT with SExtractor, GALFIT, and GIM2D can provide such statistical uncertainties, thus giving us an idea about the actual limits of the automatic tools for the surface photometry of galaxies (see Sect. 4).

The GASPHOT catalogs refer to fairly homogeneous samples in the different bands, because they have been obtained from CCD images whose exposure times have been tuned to reach almost the same photometric depth. Here, we empirically define the photometric depth as the interval $\Delta \mu$ between the brightest and faintest surface brightness level detected for each galaxy on the CCD image. The homogeneity is illustrated in Fig. 1, where the $\Delta \mu$ histograms of galaxies in four clusters (two imaged with INT and two with MPG) for the three bands are plotted as an example. Of course, higher values of $\Delta \mu$ correspond to brighter galaxies, but the figure clearly shows that the range of $\Delta \mu$ is almost the same in the three bands $(\sim 4 \div 5 \mathrm{mag})$. Therefore, we are confident that for at least the photometric depth, no statistical biases among the different filters are present in the GASPHOT parameters because of different galaxy sampling.

In Sect. 2 we recall that with the typical values of $\sigma_{\mathrm{bkg}}$ found in the WINGS-OPT $V$-band imaging, we obtain an average value of the isophotal threshold of $\mu_{\mathrm{Thr}}(V) \sim 25.7 \mathrm{mag} \operatorname{arcsec}^{-2}$. At the same signal-to-noise level, the images from SDSS in the $g$ and $r$ band reach $\mu_{\mathrm{Thr}}(g)<25.2 \mathrm{mag} \operatorname{arcsec}^{-2}$ and $\mu_{\mathrm{Thr}}(r)<$ $24.7 \mathrm{mag} \operatorname{arcsec}^{-2}$.

\section{Internal and external comparisons}

Pignatelli et al. (2006) compared the GASPHOT performances with the results from detailed, single-object surface photometry of 231 early-type galaxies published by Fasano et al. (2003) and with the surface photometry parameters obtained by Smail et al. (1997) from HST imaging of galaxies in the cluster Abell 370. They found a generally good agreement between automatic (GASPHOT) and single-object surface photometry parameters, although a large scatter and a slight tendency of underestimating the total luminosity and the effective radius of very large 

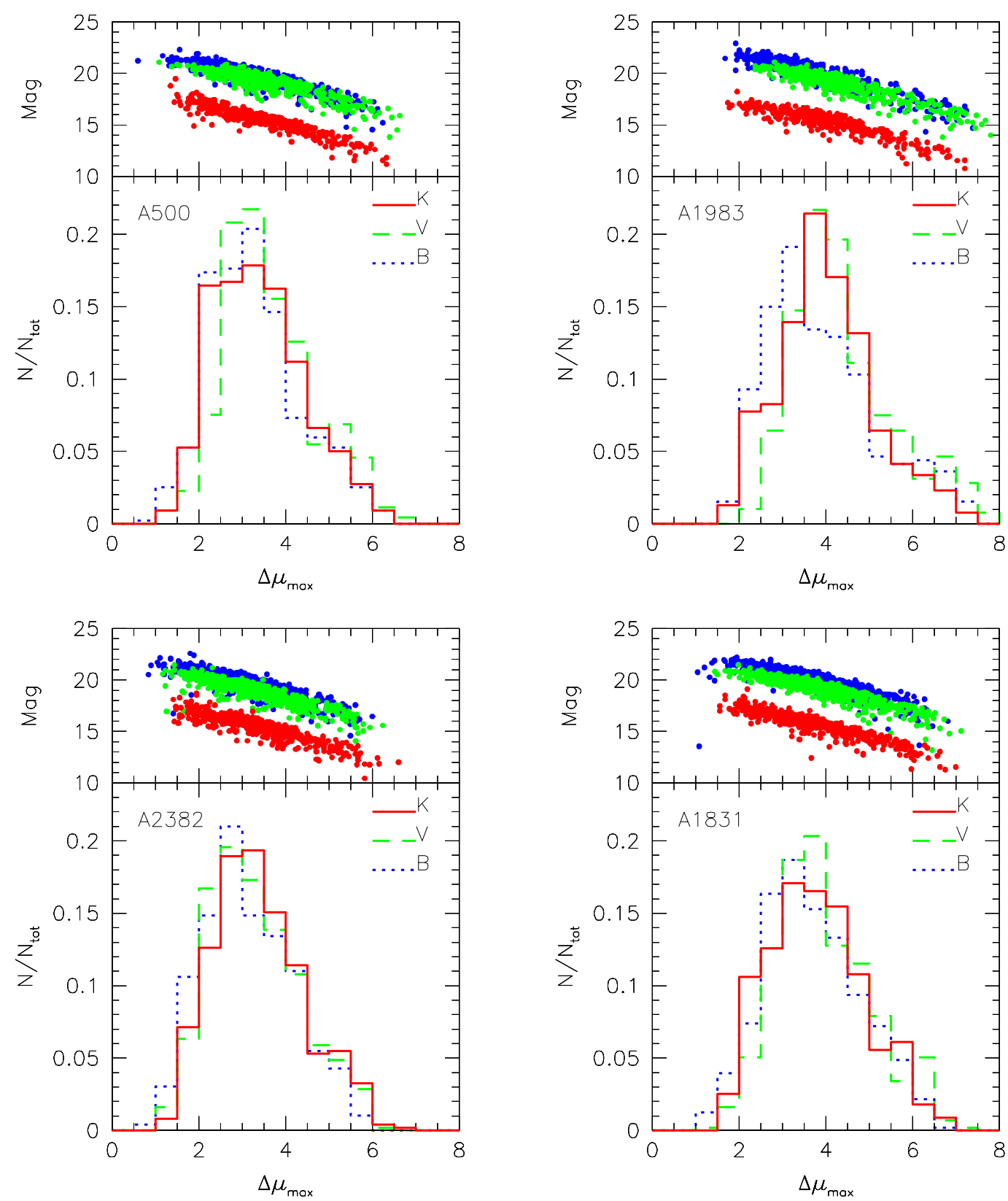

Fig. 1. Histograms of the photometric depth $\Delta \mu_{\max }$ observed in the $B, V$, and $K$ bands for four different WINGS clusters (A550, A1983, A2382, and A1831). The color legend marks the various filters. The upper panels above each histogram show the total luminosity versus $\Delta \mu_{\max }$.

galaxies seemed to be present in GASPHOT with respect to the supervised, single-object surface photometry.

In this section we test the GASPHOT results against SExtractor and GALFIT using the WINGS data, and against GIM2D using the Padova Millenium Galaxy and Group Catalog PM2GC (Calvi et al. 2011). In each comparison we use only galaxies with a SExtractor flag equal to zero and found by SExtractor as having a threshold area (above $2.5 \sigma_{\mathrm{bkg}}$ ) greater than 200 pixels. Moreover, we decided to exclude from this comparison galaxies for which GASPHOT gives $n=0.5$ or $n=8$ (boundary values for the search $n$ interval), and those for which, according to GASPHOT, the average surface brightness within the effective isophote exceeds $21.5,25.5$ and 26.5 for the $K, V$ and $B$ band, respectively. In fact, according to the quality index $Q_{\text {FLAG }}$, beyond these values the surface photometry parameters provided by GASPHOT for our galaxy samples becomes largely unreliable.

\subsection{GASPHOT vs. SExtractor}

In Fig. 2 the median total magnitude differences $\Delta \mathrm{m}$ between SExtractor and GASPHOT in the $K, V$, and $B$ band (top to bottom) are binned as a function of the best fit quantities derived by GASPHOT. They are (left to right) the absolute magnitude, the effective radius and surface brightness, the Sersic index, and the axis ratio. The error bars represent the rms uncertainty of the median values in each bin. The number of galaxies used for the comparisons are 7485, 23378 , and 22309 for the $K, V$, and $B$ band, respectively.

The most evident feature in Fig. 2 is the dependence of $\Delta \mathrm{m}$ on galaxy size in all wavebands. For large galaxies, the SExtractor magnitudes are fainter than the GASPHOT magnitudes. We have verified that this trend is particularly evident for late-type galaxies in the $B$ band. This occurs because the SExtractor deblending parameter in many cases tends 
SExtractor - GASPHOT
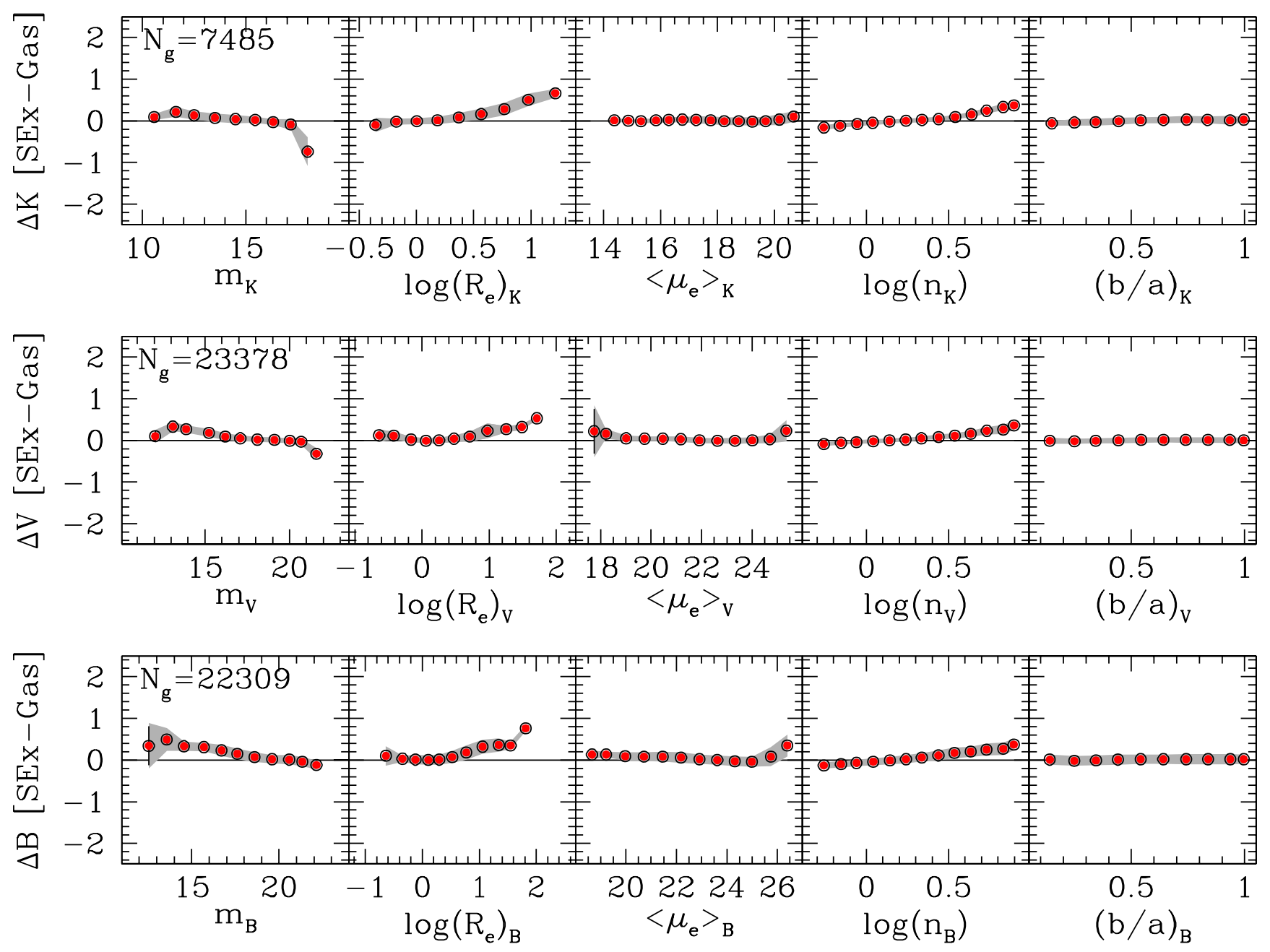

Fig. 2. Median total magnitude differences between SExtractor and GASPHOT in the $K, V$, and $B$ band (top to bottom) binned as a function of the absolute magnitude, the effective radius and surface brightness, the Sersic index, and the axis ratio derived by GASPHOT (left to right). The error bars represent the uncertainties of the median values in each bin, while the shaded bands give the semi-interquartile ranges of the deviation distributions. Both quantities are very small (comparable with the size of the points). The sizes of the samples used for the comparisons are reported in the leftmost panel for each filter.

to erroneously split large spirals into multiple, smaller objects (HII regions and other light blobs). Instead, for early-type galaxies, the magnitude difference, still present and positive, reflects the well-known inability of SExtractor to extrapolate the smoothly decreasing (high Sersic index) outer profiles of galaxies (Franceschini et al. 1998). This is confirmed by the smooth rise of $\Delta m$ as a function of the Sersic index in all wavebands (see Fig. 2), and by the attenuation of the bias when the first three ranked most luminous galaxies in each cluster are removed from the WINGS sample. The dependence of $\Delta m$ on galaxy size also determines the behavior of $\Delta m$ as a function of the luminosity. Instead, no dependence at all of $\Delta m$ on the axial ratio is found.

\subsection{GASPHOT vs. GALFIT and GIM2D}

Pignatelli et al. (2006) showed that the performances of GASPHOT on artificial galaxies are similar to those of GALFIT and GIM2D for large and regular galaxies, while for automatic surface photometry of small galaxies and blended objects, GASPHOT provides more robust results than GALFIT and
GIM2D (see Cols. 6 and 9 of Table 1 and Figs. 12 and 13 in Pignatelli et al. 2006). This is a crucial feature when dealing with blind surface photometry of huge galaxy samples.

In this section, we use real galaxies to compare the automatic surface photometry parameters from GASPHOT and GALFIT and GIM2D. For both comparisons (GALFIT-GASPHOT and GASPHOT-GIM2D) the model used to fit the galaxy luminosity profiles was the single 2D Sersic law, with constant ellipticity and position angle.

\subsubsection{Samples used for the comparisons}

For the comparison between GASPHOT and GALFIT, we used a sample of 1684 galaxies randomly extracted from the WINGSGASPHOT catalogs among those with $Q_{\text {FLAG }}=0$ and belonging to clusters whose $V$-band WINGS-OPT imaging has been obtained in good seeing conditions, with minimal PSF variation over the cluster field. On this galaxy sample GALFIT was run taking as initial guess for the parameters the $V$ band 


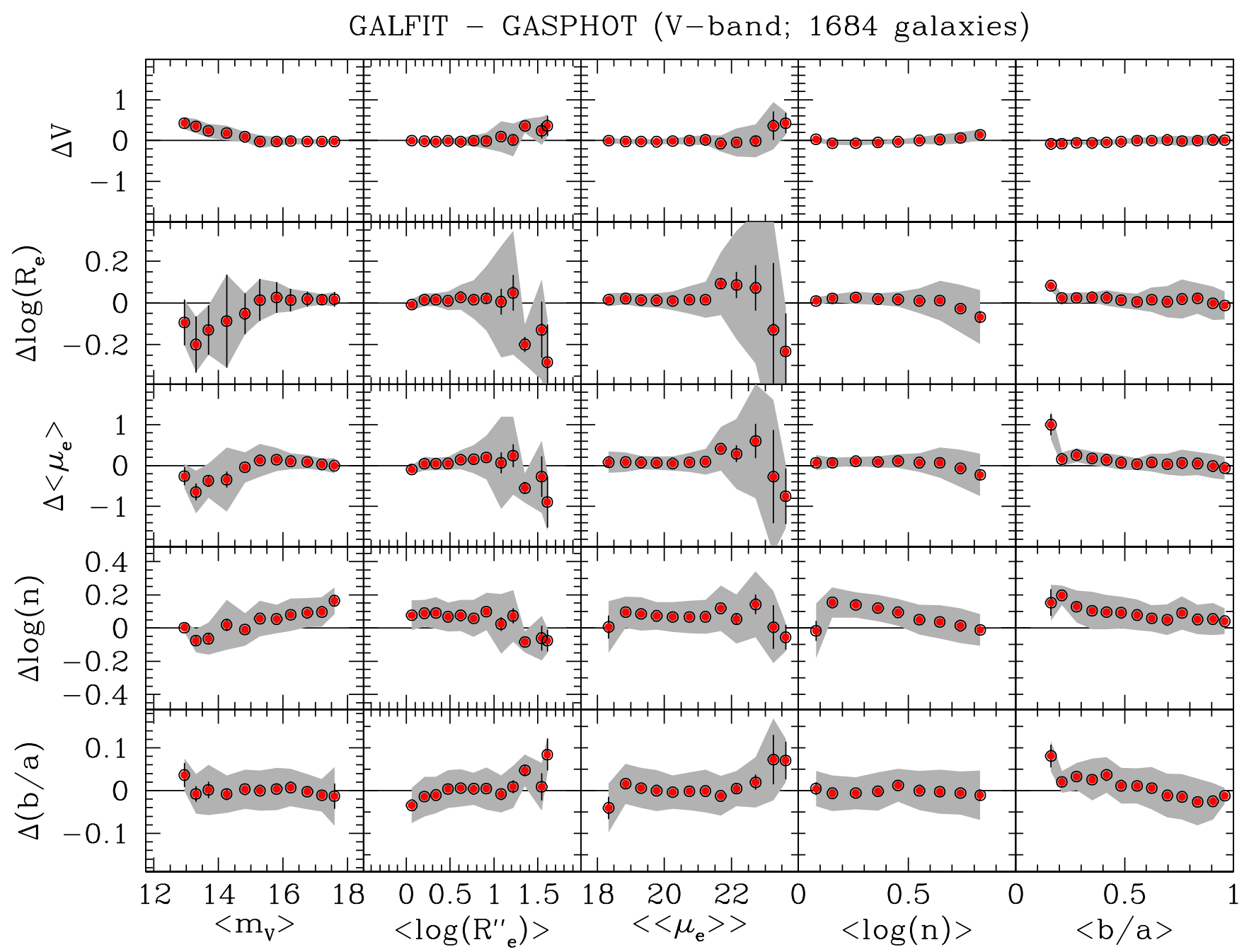

Fig. 3. Comparison between the results of GASPHOT and GALFIT surface photometry for 1684 galaxies randomly extracted from the WINGS $V$-band catalogs. The comparison is made for apparent total magnitude, effective radius in arcseconds, effective average surface brightness, Sersic index, and axis ratio. The parameters in abscissa are averaged between the tools under comparison, while the differences between the values found by the tools are reported for each parameter on the ordinate, binned over the whole set of parameters. As in Fig. 2, the error bars represent the uncertainties of the median values of the differences in each bin, while the shaded bands give the semi-interquartile ranges of the deviation distributions.

photometric and geometric quantities provided by the WINGSOPT SExtractor catalogs.

The CPU time required by GALFIT to produce the surface photometry parameters of a single galaxy is (on average) about twice longer than in the case of GASPHOT. This is probably because GALFIT has to handle the whole set of pixels belonging to each galaxy, while GASPHOT only deals with the major- and minor- axis growth curves.

We compare GIM2D and GASPHOT using galaxies in a sub-sample of the Millennium Galaxy Catalog (MGC; Liske et al. 2003; Cross et al. 2004). The MGC survey is based on $B$ band imaging taken with the WFC camera of the Isaac Newton Telescope (the same as used by WINGS in the northern hemisphere; pixel size of 0.333 arcsec) along an equatorial strip covering an area of $\sim 37.5 \mathrm{deg}^{2}$. The MGC images reach an isophotal detection limit of $26.0 \mathrm{mag} \operatorname{arcsec}^{-2}$.

The GIM2D data come from three different works based on the MGC imaging: the surface photometry by Allen et al. (2006), that of the New York University Value Added Catalogue (NYUVAC Blanton et al. 2005), and that from the SLOAN DR7 data (Simard et al. 2011).
We have obtained GASPHOT surface photometry for a sample of galaxies in the PM2GC (Calvi et al. 2011), which is a galaxy catalog extracted from the MGC and representative of the general field population in the local Universe $(0.04 \leq z \leq 0.1)$.

A preliminary comparison between GASPHOT and GIM2D was presented by Poggianti et al. (2013a) for 618 galaxies in common between the PM2GC and MGC surveys. They found that the agreement between GASPHOT and GIM2D is generally good, with a tendency for the GASPHOT radii to be slightly larger than the others. The median difference between the effective radii $R_{\mathrm{e}}$ is about $0.03 \pm 0.04$ dex with respect to the data of Allen et al. (2006), $0.03 \pm 0.06$ dex with respect to the NYUVAC, and $-0.01 \pm 0.04$ dex with respect to Simard et al. (2011).

We compare here GASPHOT and GIM2D using an extended sample of 2581 galaxies in common between the PM2GC and MGC surveys. For GIM2D we used the literature data from the same sources. It was therefore not possible to test the CPU time of GIM2D for a fit of a single galaxy. However, we recall that GIM2D was found by Pignatelli et al. (2006) to be significantly 


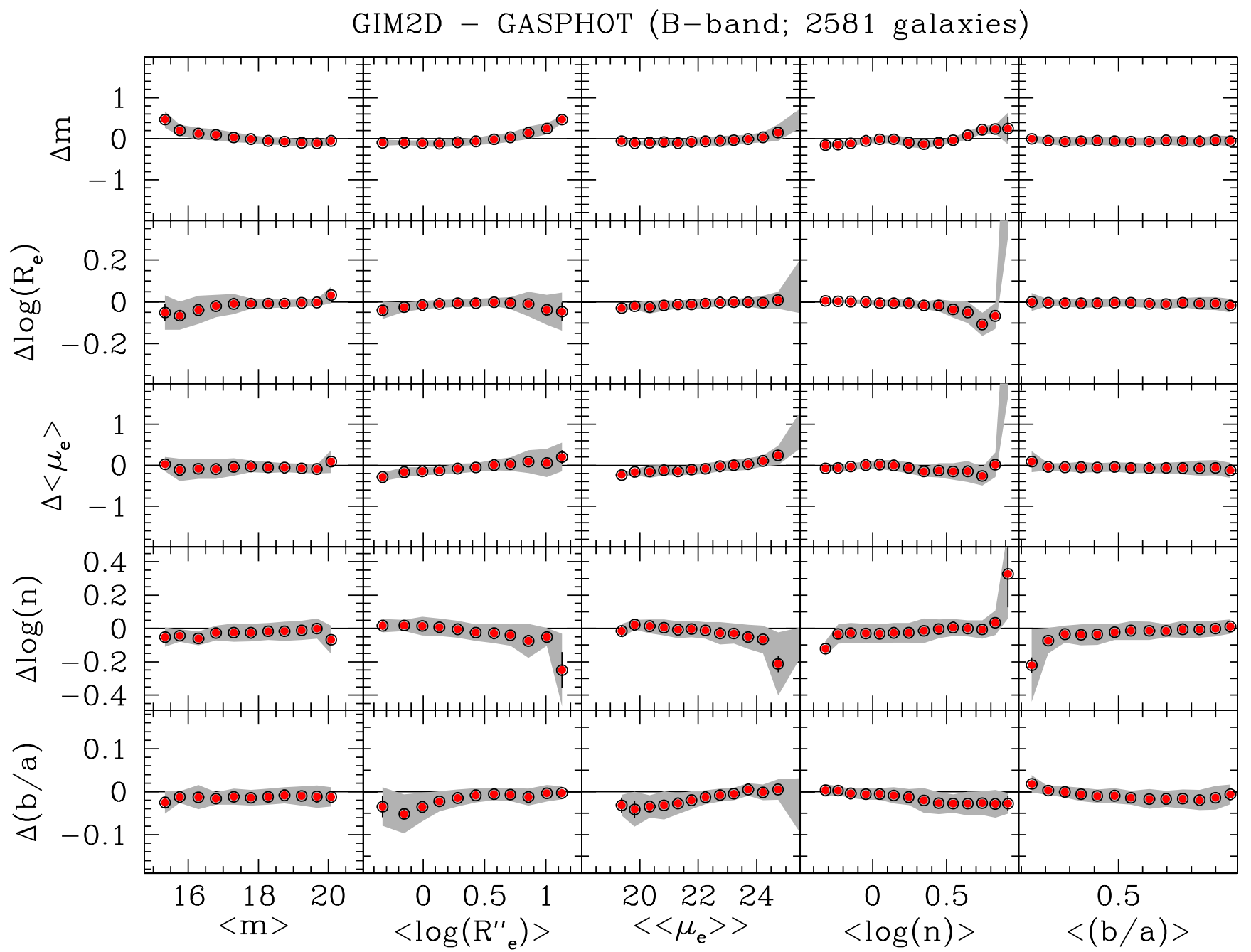

Fig. 4. Same as Fig. 3, but for the GIM2D - GASPHOT comparison ( $B$ band) on the sample of 2581 galaxies in common between the surveys PM2GC and MGC (Allen et al. 2006).

more expensive in terms of CPU time with respect to both GASPHOT and GALFIT.

To discuss the features of GASPHOT, GALFIT, and GIM2D in detail is beyond the scope of this paper. They are provided in previous researches. Neither do we aim here to propose a ranking of goodness for the three tools. The comparison we perform in this section is only intended to provide an estimate of the uncertainties of the surface photometry parameters obtained with automatic tools. Still, it is useful to summarize here how the data used for the comparison have been obtained, in particular in the matter of the sky background subtraction, the PSF modeling, and the best-fit scheme.

\subsubsection{Sky subtraction}

The WINGS images used here for both GASPHOT and GALFIT surface photometry have already been sky subtracted using the procedure described in Varela et al. (2009, see also Sect. 2).

GASPHOT can use the sky level $I_{\mathrm{bkg}}$ as a free modelparameter of the best-fitting procedure. However, since this might be dangerous, particularly for blended objects, the user can limit the range of variation for this parameter when one is confident that a careful sky subtraction has already been performed on the images. This is our case, and we allowed $I_{\mathrm{bkg}}$ to vary of $1.8 \times \sigma_{\mathrm{bkg}}$ at most.

GALFIT can also consider the sky level $I_{\mathrm{bkg}}$ as a free modelparameter of the best-fitting procedure. However, since no restricted range of $I_{\mathrm{bkg}}$ variation is allowed in GALFIT, we preferred not to include it among the free parameters and fixed its value at zero.

The treatment of the background subtraction is not homogeneous in the literature sources of the GIM2D surface photometry data used for our comparison (Blanton et al. 2005; Allen et al. 2006; Simard et al. 2011). It ranges from a crude SExtractor estimate of the global sky level to a more accurate determination, adopting for each galaxy a minimum distance of background pixels from object pixels, defined by segmentation mask images.

\subsubsection{PSF modeling}

GASPHOT automatically extracts the profiles of the stars, models the variation of the FWHM through the field with a 2D polynomial of user-defined degree, and combines the PSF profiles, after rescaling them according to the space-varying model obtained previously. Finally, a multi-Gaussian function is used to perform the $\chi^{2}$ best-fit of the average PSF profile. The Sersic 
profiles are then convolved with a PSF whose Gaussian coefficients depend on the position of the galaxy in the frame.

Both GALFIT and GIM2D assume the user to be able to provide a suitable PSF for each galaxy, both from star images or by functional form. When running GALFIT on our sample of WINGS galaxies, we decided to adopt a single average PSF image for each cluster, we therefore cannot account for minor PSF differences over the image. However, because of the previously outlined choice of the WINGS imaging for the GASPHOT-GALFIT comparison, this probably does not significantly contribute to worsen the results.

Again for GIM2D the determination of the PSF is not homogeneous in the literature data used for our comparison; it ranges from a unique PSF for all galaxies in a given image to a more sophisticated (space-varying) treatment, and from a simple Gaussian profile to a more complex (functional or user-defined) form.

\subsubsection{Best-fitting}

GALFIT uses the Levenberg-Marquardt downhill-gradient method to derive the best fit. An error map image is automatically produced by the software. At each pixel position the Poisson error is evaluated on the basis of the gain and readnoise parameters contained in the image header. Good fits can be obtained only when the error map is well known and used as weighting image.

The Metropolis best-fit algorithm used by GIM2D (Metropolis et al. 1953; Saha \& Williams 1994) is more CPU expensive than the Levenberg-Marquardt downhillgradient method used by GALFIT. However, it is claimed to be particularly suited to explore an $n$-dimensional parameter space (with $n$ possibly larger than 10 ) and has a very complicated topology with local minima at low S/N ratios. As in GALFIT, a noise map is used to weight pixels.

After producing the isophotes of all galaxies together (see Sect. 3), GASPHOT performs for each galaxy a simultaneous Levenberg-Marquardt $\chi^{2}$ best-fit of the major- and minor-axis growth curves with a 2D Sersic law, convolved with the proper PSF. Each point of the growth curves is weighted according to the statistical uncertainties on both the integrated isophotal magnitude and the radius (pixellation). Compared with the $\mathrm{S} / \mathrm{N}$ driven, pixel-by-pixel weighting commonly used in the genuine $2 \mathrm{D}$ tools, this procedure tends to overweight the outer part of the profiles, because is is less sensitive to the high $\mathrm{S} / \mathrm{N}$ peculiar features (dust-lanes, cores, small bars and rings, etc.) that often affect the innermost galaxy body. This probably makes GASPHOT particularly useful when dealing with large/huge galaxy samples, for which detailed, single-object (visually supervised) modeling must be sacrificed to the advantage of speed and robustness.

\subsubsection{Results}

Figures 3 and 4 illustrate the differences between surface photometry parameters for the GALFIT-GASPHOT and GIM2DGASPHOT comparisons. The surface photometry parameters used for the comparisons are the apparent total magnitude, the effective radius in arcseconds, the effective average surface brightness, the Sersic index, and the axis ratio. Since we cannot a priori assume one of the tools to give more reliable results than the others, the above parameters in abscissa are averaged between the tools under comparison in both figures. The
Table 2. Global median values and rms of the differences (GALFITGASPHOT) and (GIM2D-GASPHOT) are reported for the apparent magnitude ( $V$ and $B$ band, for the first and second comparison respectively), the effective radius and surface brightness, the Sersic index, and the axis ratio.

\begin{tabular}{|c|c|c|c|c|c|}
\hline \multicolumn{6}{|c|}{ GALFIT-GASPHOT } \\
\hline & $\Delta m$ & $\Delta \log R_{\mathrm{e}}$ & $\Delta\langle\mu\rangle_{\mathrm{e}}$ & $\Delta \log n$ & $\Delta(b / a)$ \\
\hline$\langle\Delta\rangle$ & -0.020 & 0.016 & 0.086 & 0.077 & -0.001 \\
\hline$\sigma_{\Delta}$ & 0.123 & 0.077 & 0.320 & 0.116 & 0.067 \\
\hline \multicolumn{6}{|c|}{ GIM2D-GASPHOT } \\
\hline$\langle\Delta\rangle$ & -0.068 & -0.006 & -0.049 & -0.014 & -0.012 \\
\hline$\sigma_{\Delta}$ & 0.125 & 0.030 & 0.187 & 0.080 & 0.028 \\
\hline
\end{tabular}

differences between the values found by the tools are reported for each parameter on the ordinate, binned over the whole set of parameters. Moreover, the error bars in the figures represent the $1 \sigma$ uncertainties of the median values of the differences in each bin, while the shaded bands give the semi-interquartile ranges of the deviation distributions.

In Table 2 the global median values and rms of the differences (GALFIT-GASPHOT) and (GIM2D-GASPHOT) are reported for the same surface photometry parameters as used in Figs. 3 and 4.

Figures and Table 2 show that the general agreement is better and the scatter smaller for the GIM2D-GASPHOT than for the GALFIT-GASPHOT differences. This cannot be due to the different size of the two samples (2581 vs.1684), since a larger scatter in the GALFIT-GASPHOT plots is found even considering only early-type galaxies (plot not shown), for which the sample size is greater for the GALFIT-GASPHOT than for the GIM2D-GASPHOT comparison (1491 vs.1126). Thus, we should either guess the intrinsic uncertainty to be larger for GALFIT than for GIM2D or, alternatively, we could speculate about a sort of environment-driven, additional scatter, that makes the surface photometry of galaxies less reliable in the cluster (GALFIT) than in the general field (GIM2D) environment.

The plots also show the dependence of the scaling quantity differences $\left(\Delta m, \Delta \log \left(R_{\mathrm{e}}\right)\right.$ and $\left.\Delta\langle\mu\rangle_{\mathrm{e}}\right)$ on the galaxy scaling parameters themselves. In particular, for large and bright galaxies, GASPHOT seems to produce best-fit galaxy models brighter and larger than GALFIT and (just marginally!) GIM2D. Since the same is true for the SExtractor-GASPHOT comparison (see Fig. 2), we might be induced to conclude that this behaviour is caused by some size-drived bias of the GASPHOT surface photometry (although an opposite tendency has been noted before; see the first sentence of Sect. 4). However, we ruled out this conclusion on the basis of the following arguments:

(i) the inability of SExtractor to extrapolate the smoothly decreasing (high Sersic index) outer profiles of bright galaxies is probably responsible for the size-driven magnitude differences between SExtractor and GASPHOT, particularly for shallower imaging, as in the case of the $B$ and (even more) $K$ band WINGS imaging;

(ii) the agreement with the GIM2D photometry is better than for the other comparisons. In particular, the size-driven differences are much less evident, and some of the systematic differences present in the GALFIT-GASPHOT comparison 


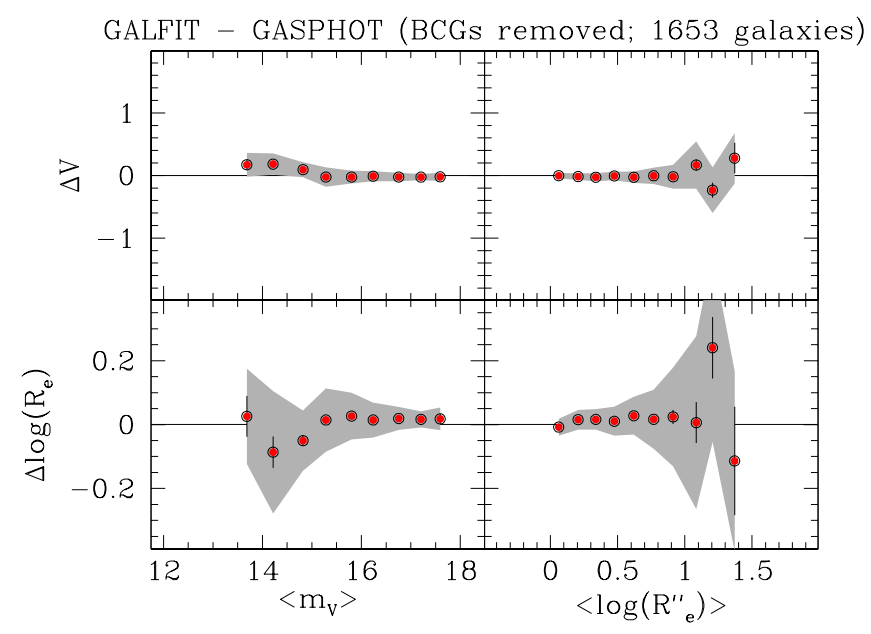

Fig. 5. Comparison of the apparent magnitude and effective radius between GASPHOT and GALFIT for the sample of Fig. 3, after removing the BCGs. In this case the size-driven bias visible in Fig. 3 is much lower or even absent (see text).

disappear, or even behave in the opposite direction (see, for instance, $\Delta \log (n)$ and $\left.\Delta\langle\mu\rangle_{\mathrm{e}}\right)$;

(iii) a natural attitude of GASPHOT to well represent the outer luminosity profiles of large (halo-equipped) galaxies is expected, because of the GASPHOT tendency to overweight the outer regions of galaxies with respect to the other tools (see Sect. 4.2.4). The most evident size-driven differences between GALFIT and GASPHOT are probably due to this different weight allocation, which is particularly effective for large, luminous galaxies. In this context, it is interesting to note that these systematic differences almost disappear when we exclude the BCGs from the sample (Fig. 5).

\subsubsection{Final remarks}

In general, the systematic differences among the parameters provided by different surface photometry tools are expected because of the different surface photometry techniques adopted. As mentioned in Sect. 3, the GASPHOT algorithm is a hybrid between the 1D (equivalent luminosity profile fitting) and 2D (full-image fitting) approach.

Of course, advantages and disadvantages can be found for both approaches. As a general rule, even if the 1D technique is unable to model either the inner (seeing-affected) regions of flattened galaxies or possible misalignments between different galaxy components, it has the advantage of being less sensitive to the peculiar features of real galaxies, since the elliptical isophotes are averaged over a large number of pixels and their parameters (coordinates of the center, semi-major axis, ellipticity, and position angle) can be derived even for very noisy and irregular shapes. In contrast the 2D approach is fully equipped to handle the above mentioned issues (convolution of seeingaffected regions of flattened galaxies and modeling of misalignment between different galaxy components), but its sensitivity to the galaxy peculiar features makes its blind (not supervised) application to large galaxy samples dangerous, because it might produce unrealistic results for a fraction of the sample. Roughly speaking, the 1D approach is more robust, because it provides reasonable results even in critical situations, while the 2D approach is suitable for supervised, detailed modeling of wellsampled objects, even for multicomponent structures and in the very inner region of galaxies (Haussler et al. 2007; Pignatelli et al. 2006; Lotz et al. 2006; Blanton et al. 2003; Bershady et al. 2000).

GASPHOT tries to exploit the robustness of the 1D fitting technique, and at the same time retain the capability of dealing with PSF convolution in the innermost regions typical of the 2D approach. GASPHOT substantially reduces the amount of interaction for the user and (mainly working in blind mode) is able to provide robust estimates of the relevant global parameters for the hundreds of galaxies typically found in wide and deep-field images.

\subsection{GASPHOT parameters in different bands}

After comparing the results of GASPHOT with the alternative tools GALFIT and GIM2D, in this section we compare the structural parameters obtained by GASPHOT in the $B, V$, and $K$ band.

In Fig. 6 the various structural parameters obtained by GASPHOT in the different bands are compared as a function of the parameters themselves, averaged between the filters under comparison. In particular, the upper panels refer to the $B$ vs. $V$ band comparison, while the lower panels illustrate the $V$ vs. $K$ band comparison. In the plots comparing the total magnitude and the effective surface brightness, the $\Delta m$ and $\Delta\langle\mu\rangle_{\mathrm{e}}$ differences are normalized by subtracting the average colors to emphasize the trends of the relations, that is the total magnitude differences, averaged over the whole samples.

For all the photometric and structural parameters, the agreement between the $B$ and $V$ band is fairly good. Moreover, no significant trends are found, apart from a slight tendency of faint, small galaxies and a more marked tendency of high Sersic index galaxies to be redder. Instead, in the plots comparing the $V$ and $K$ band GASPHOT parameters, the general agreement is poorer than in the previous case. In addition, various offsets and trends are clearly visible. In particular, compared with the $V$ band, the structure of galaxies in the $K$ band shows (on average) a higher Sersic index, a smaller effective radius, and a brighter effective surface brightness, even after removing the global galaxy color. In addition, the tendency (already mentioned for the $B$ vs. $V$ band comparison) of high Sersic index galaxies to be redder becomes much more evident in the $V$ vs. $K$ band. All these facts are consistent with the picture proposed by D'Onofrio et al. (2011, see also D'Onofrio et al. 2013b), in which at increasing stellar mass (luminosity), early-type galaxies become on average older (redder) and more centrally concentrated (higher Sersic index). The dependence of the effective radius on the waveband has also been discussed in Poggianti et al. (2013a).

\section{5. $V$-band structural parameters of galaxies in the WINGS clusters}

In this section we briefly illustrate a few statistical properties of the structural parameters of WINGS galaxies and some relations among them. To produce the plots presented in this section, the galaxy sample described in Sect. 4 was additionally restricted to only include the spectroscopic members of WINGS clusters for which we have $V$-band GASPHOT surface photometry (3131 galaxies). Relying on the morphological classification obtained by MORPHOT, we divided this sample into four broad morphological types: (i) elliptical galaxies $(T=-5,952 \mathrm{ob}-$ jects); (ii) S0 galaxies ( $-5<T \leq 0,1478$ objects); (iii) early spirals $(0<T<5,593$ objects), and (iv) late spirals $(T \geq 5$, 108 objects). 
GASPHOT(B)-GASPHOT(V) (21452 galaxies)

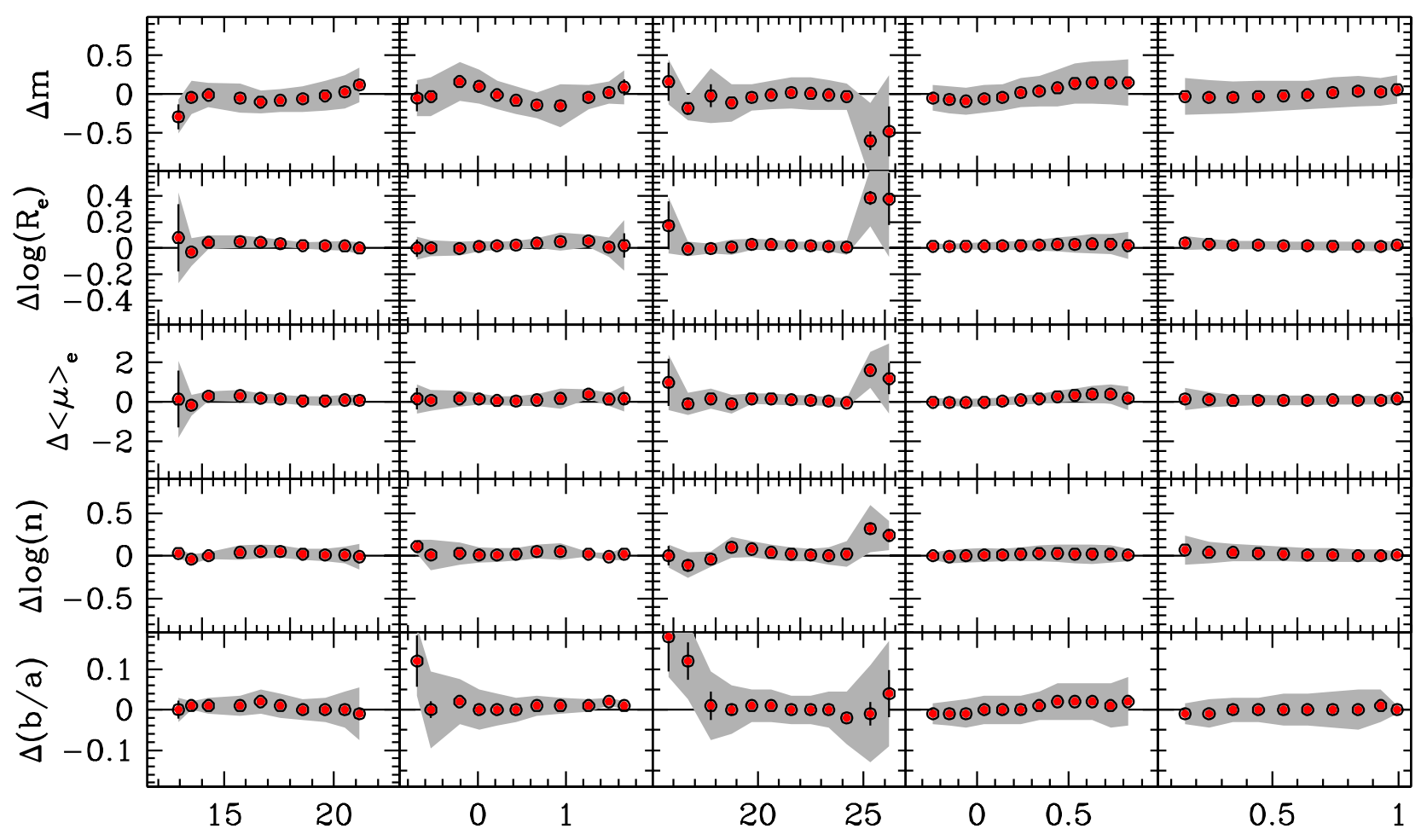

GASPHOT(V)-GASPHOT(K) (5124 galaxies)

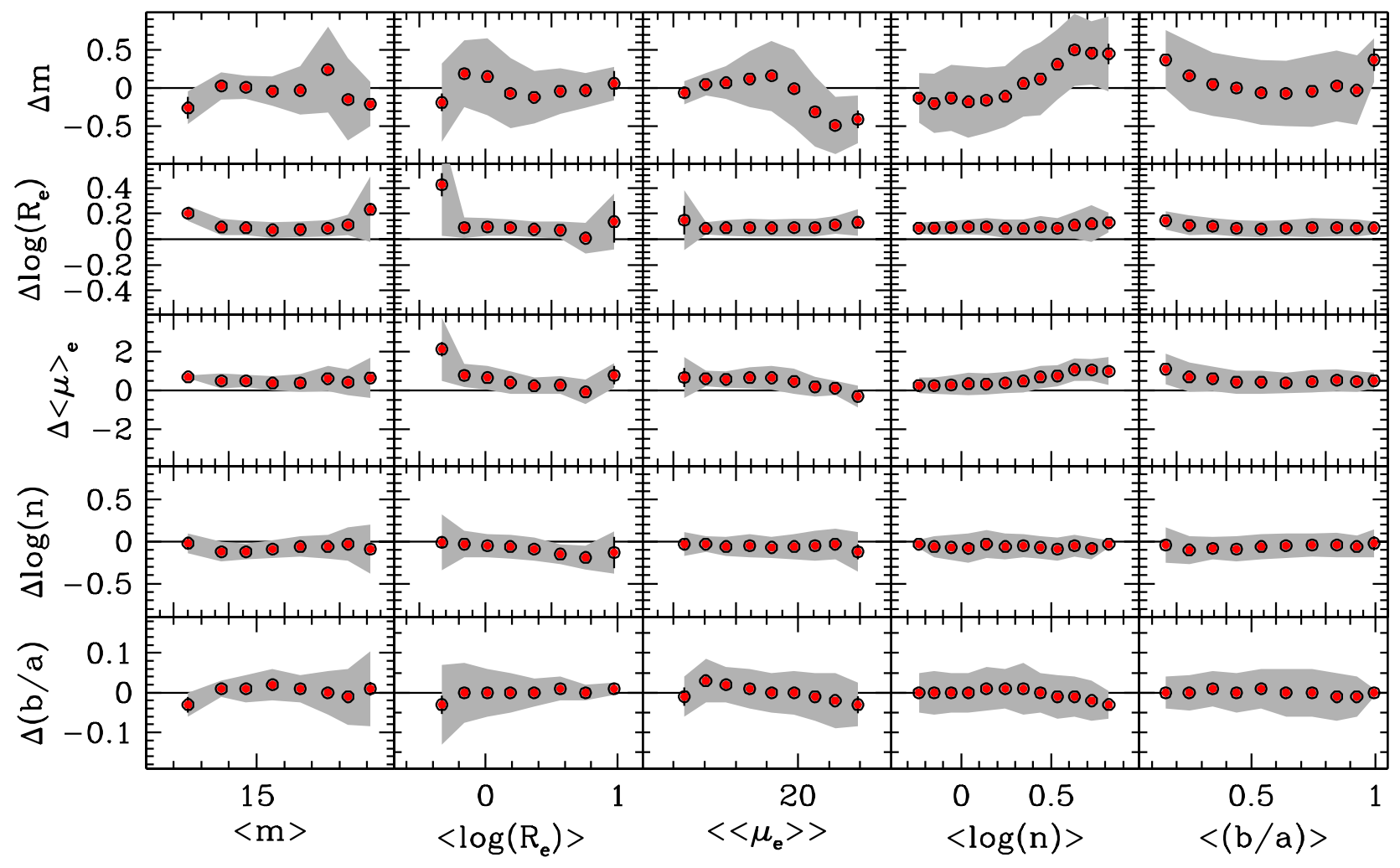

Fig. 6. Comparison among various structural parameters obtained by GASPHOT in the different bands. The upper panels refer to the $B$ vs. $V$ band comparison, while the lower panels illustrate the $V$ vs. $K$ band comparison. The differences between parameters in different bands are plotted vs. the parameters themselves, averaged between the filters under comparison. The $\Delta m$ and $\Delta\langle\mu\rangle_{\mathrm{e}}$ differences are normalized by removing the average colors (see text). As in the previous figures, the error bars represent the uncertainties of the median values of the differences in each bin, while the shaded bands give the semi-interquartile ranges of the deviation distributions. 


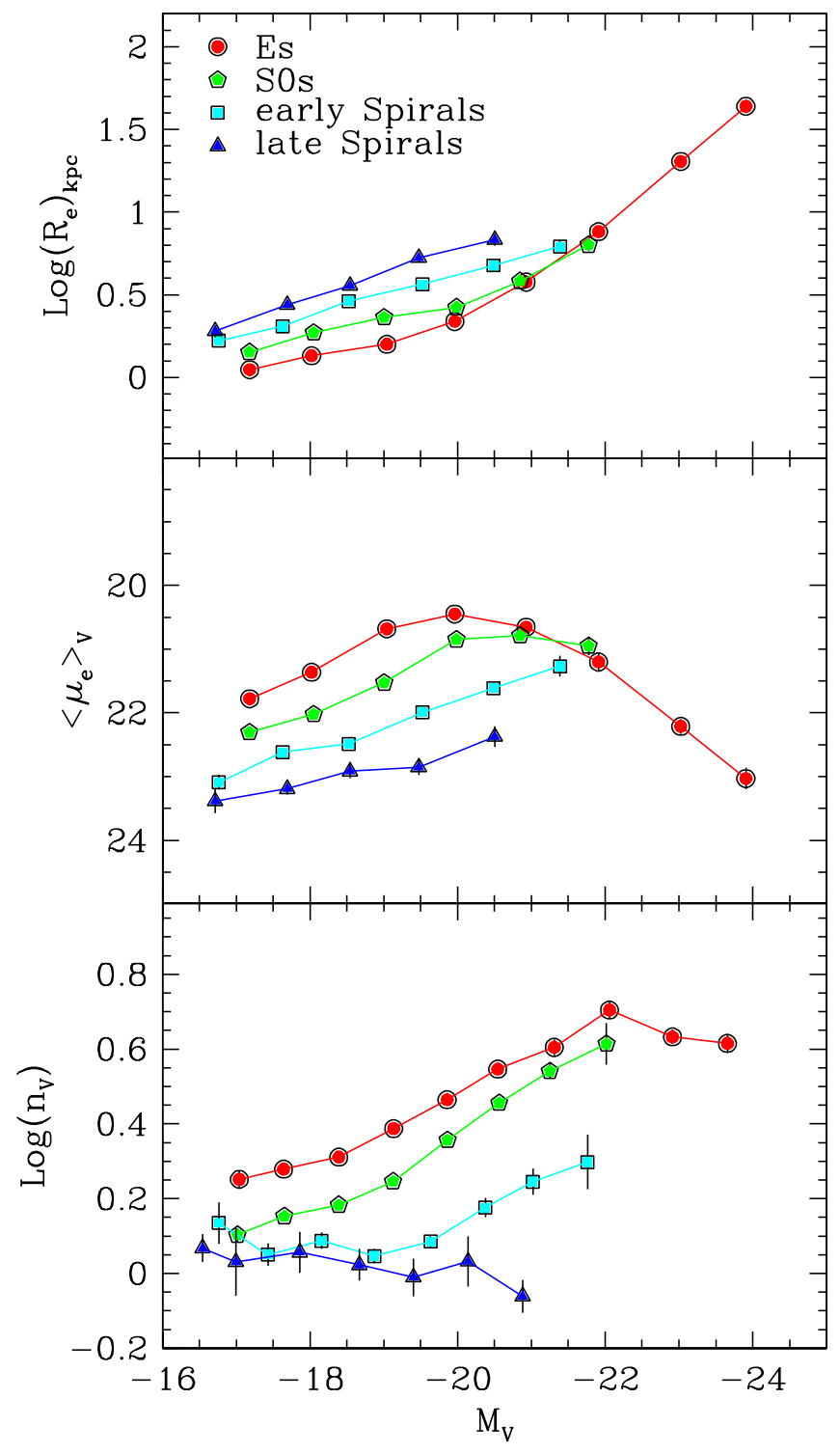

Fig. 7. Effective radius (upper panel), mean surface brightness, and Sersic index (lower panel) obtained by GASPHOT for the WINGS galaxies in the $V$ band as a function of the absolute magnitude for the four, broad morphological types. The mean values of the structural parameters are binned over the absolute magnitude. Ellipticals, S0s, early and late spirals are represented by circles, pentagons, squares, and triangles (red, green, cyan and blue).

Figure 7 illustrates how the structural parameters obtained by GASPHOT for the WINGS galaxies in the $V$ band, depend on the absolute magnitude for the four, broad morphological types. It shows that for a given absolute magnitude, the later the morphological type, the lower the Sersic index and the larger the effective radius and surface brightness. Moreover, at increasing total luminosity, the effective surface brightness decreases (with the notable exception of the brightest ellipticals), while both the Sersic index and the effective radius increase, with the exception of the Sersic index of late spirals, which slightly decreases at increasing the total luminosity. Similar trends have been found in the recent literature (Cebrián \& Trujillo 2014; Bernardi et al. 2014).

The brightest ellipticals show a strong overturning of the surface brightness trend and a less pronounced (but still clear) break of the Sersic index trend. The last feature, absent in the classical relation discovered by Caon et al. (1993) for the Virgo cluster galaxies, has likely emerged here because of the much more robust statistics on the BCGs. These features, together with the marked increase of their size at increasing the total luminosity, are consistent with the picture of BCGs as a separate class of objects, distinct from normal Ellipticals and dominated by the cD galaxies (Capaccioli et al. 1992; Fasano et al. 2010).

In Fig. 8 the distributions of the same structural parameters of Fig. 7 (in addition to the ellipticity), normalized to the area subtended by the histograms, are reported for the four broad morphological types. The continuity of the distributions when moving from elliptical galaxies towards later types is remarkable. In this context, the ellipticity distributions constitute an exception, to some extent because the flattening distribution of ellipticals is quite different from those of any other morphological type, in agreement with previous analyses (Fasano \& Vio 1991; Fasano et al. 1993, 2010).

Finally, in Fig. 9 we plot the galaxies of the four broad morphological types, onto the $\langle\mu\rangle_{\mathrm{e}}-\log \left(R_{\mathrm{e}}\right)$ plane, in turn divided in two subsamples, according to the Sersic index (full dots: $n>3$; crosses: $n \leq 3$ ). The well-known Kormendy relation (KR; Kormendy 1977; Capaccioli et al. 1992) seems to hold only for high Sersic index elliptical galaxies. It looks much less evident (and with a different slope) for low Sersic index ellipticals and S0s, while it is not present at all for spiral galaxies, at least when a single Sersic law is used to represent their luminosity profiles. The large scatter of the KR even for ellipticals with $n>3$ is reduced when we consider only galaxies with an isophotal area larger than $10^{3}$ pixels at $2.5 \sigma_{\mathrm{bkg}}$ (full dots in Fig. 9).

\section{Conclusions}

We have presented the $B, V$ and $K$ band structural parameters of the WINGS cluster galaxies with an isophotal area larger than 200 pixels at the threshold of $2.5 \sigma_{\mathrm{bkg}}$ in each band. The surface photometry was obtained by means of the automatic tool GASPHOT, which performs a simultaneous $\chi^{2}$ best-fitting of the major- and minor-axis growth curves of galaxies using a single Sersic law convolved with a space-varying PSF. For each cluster of the WINGS survey, GASPHOT produced catalogs for the three photometric bands. The catalogs, available at the CDS, provide for each galaxy the WINGS identification, the coordinates of the galaxy center, the total magnitude, the effective radius, the Sersic index, the axis ratios, and a quality index flag related to the goodness of each fit.

Thanks to this database, several thousand galaxies in nearby clusters now have reliably characterized structural properties. These data have been already used in many papers of the WINGS series (see, e.g., D'Onofrio et al. 2011, 2013a,b; Poggianti et al. 2009, 2013a,b,c; Fasano et al. 2010, 2012; Vulcani et al. 2011a,b, 2012; Bettoni et al. 2011; Ascaso et al. 2011; Valentinuzzi et al. 2010b,a). The WINGS database, including the GASPHOT catalogs, is useful for comparing the results of high-redshift surveys with the zero-point reference frame of objects at low redshifts. A complete description of this database can be found in Moretti et al. (2014).

The GASPHOT output was tested through direct comparisons against SExtractor (only for total magnitudes), GALFIT, and GIM2D. The comparison with GALFIT was made in the $V$ band using a subset of the WINGS data, while that with GIM2D made use of the PM2GC data in the $B$ band. The agreement among GASPHOT and these photometric tools was 

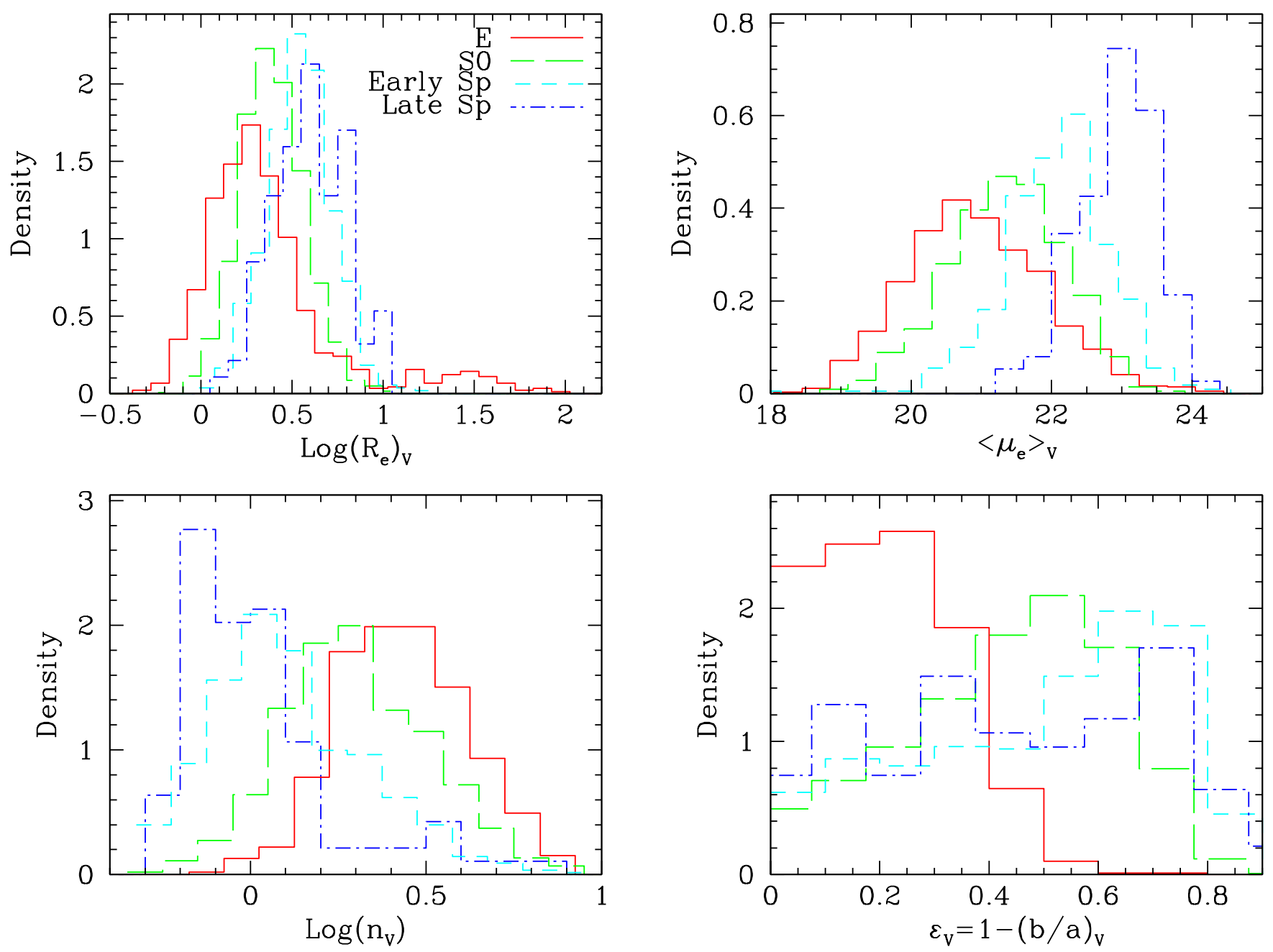

Fig. 8. Distribution of the $V$ band structural parameters derived by GASPHOT for the four broad morphological types. The distributions are normalized to the area subtended by the histograms. Ellipticals, S0s, early and late spirals are respectively indicated by solid, long-dashed, shortdashed and dot-dashed lines (red, green, cyan and blue).

generally good, apart from the tendency of SExtractor to progressively underestimate the luminosity of large or bright galaxies with respect to GASPHOT (see Sect. 4.1). A similar (less pronounced) size-driven bias seems to be present also when comparing the total magnitudes from GASPHOT with those coming from GALFIT. However, this bias disappears when we removed the BCGs from the comparison sample. This is most likely due to the GASPHOT tendency of overweighting the outer regions of galaxies with respect to the other tools; this tendency is particularly effective for large, luminous galaxies (see Sect. 4.2.4).

The uncertainties of the surface photometry parameters can be estimated from the scatter of the differences among the values of the parameters obtained using different tools. In particular, the average uncertainty of the total luminosity is $\sim 12 \%$ for both the GALFIT-GASPHOT and GIM2D-GASPHOT differences. Instead, the average uncertainties of the other surface photometry parameters are significantly lower for the GIM2DGASPHOT than for the GALFIT-GASPHOT differences. They range from $7 \%$ to $20 \%$ for the effective radius and from $20 \%$ to $30 \%$ for both the effective surface brightness and the Sersic index. These uncertainties are most likely due to various effects, as already discussed by Pignatelli et al. (2006) and Haussler et al. (2007). Among them we stress the intrinsically different
1D approach followed by GASPHOT with respect to the 2D approach followed by GALFIT and GIM2D. We showed that these two methods have a different sensitivity to the peculiar features of galaxies and behave differently in weighting the various (inner and outer) galaxy regions.

The comparison among GASPHOT results in different wavebands agrees fairly well. Moreover, the trends observed in the colors (especially the $V-K$ ) as a function of $\langle\mu\rangle_{\mathrm{e}}$ and of the Sersic index are consistent with the picture proposed by D'Onofrio et al. (2011, see also D'Onofrio et al. 2013b), in which at increasing stellar mass (luminosity), early-type galaxies become on average older (redder) and more centrally concentrated (higher Sersic index).

In conclusion, GASPHOT has proven to be effective in performing automatic, blind surface photometry of galaxies in the intermediate and low spatial resolution regime (ground-based, wide-field imaging at low redshift or space-based imaging at intermediate redshift). In these cases, GASPHOT is able to quickly provide robust structural parameters of large galaxy samples. We plan to use GASPHOT to obtain the surface photometry of galaxies in the wide-field images of many southern clusters taken with OmegaCam at the VST in the framework of the WINGS survey. 
M. D'Onofrio et al.: Surface photometry of WINGS galaxies with GASPHOT

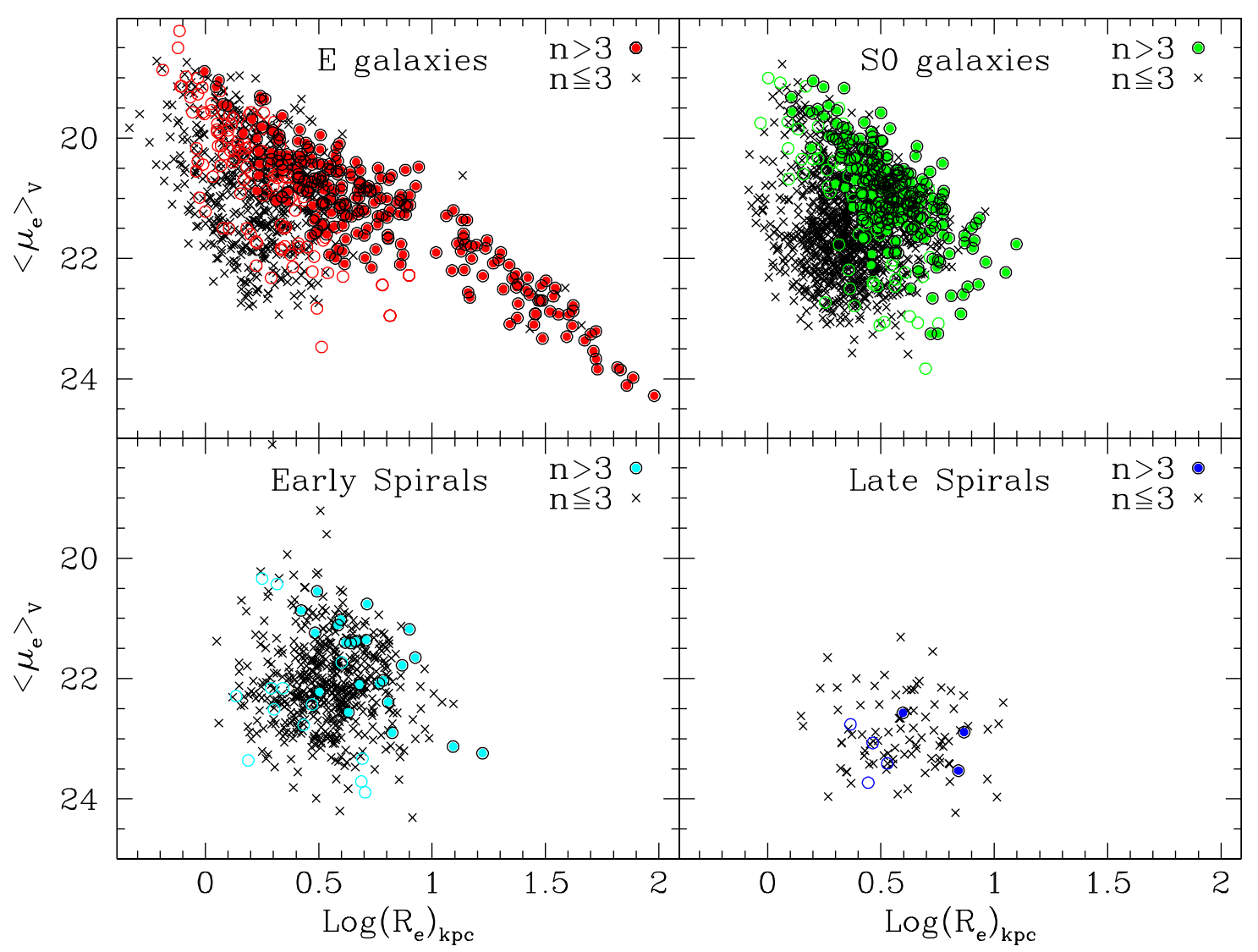

Fig. 9. The $\langle\mu\rangle_{\mathrm{e}}-\log \left(R_{\mathrm{e}}\right)$ plane for the galaxies of the four broad morphological types and for two ranges of Sersic index (circles: $n>3$; crosses: $n \leq 3$ ). Open circles mark galaxies with $n>3$ and an isophotal area smaller than $10^{3}$ pixels at $2.5 \sigma_{\mathrm{bkg}}$

Acknowledgements. The authors thank Ignacio Trujillo for his help in the analysis of the WINGS galaxies with GALFIT.

\section{References}

Allen, P., Driver, S., Graham, A., et al. 2006, MNRAS, 371, 2 Ascaso, B., Aguerri, J. A. L., Varela, J., et al. 2011, ApJ, 726, 69 Bernardi, M., Meert, A., Vikram, V., et al. 2014, MNRAS, 443, 874 Bershady, M. A., Jangren, A., \& Conselice, C. J. 2000, AJ, 119, 2645 Bertin, E., \& Arnouts, S. 1996, A\&AS, 177, 393

Bettoni, D., Kjærgaard, P., Milvang-Jensen, B., et al. 2011, Astron. Nachr., 332, 299

Blanton, M. R., Hogg, D. W., Bahcall, N. A., et al. 2003, ApJ, 592, 819

Blanton, M., Schlegel, D., Strauss, M., et al. 2005, AJ, 129, 2562

Calvi, R., Poggianti, B., \& Vulcani, B. 2011, MNRAS, 416, 727

Caon, N., Capaccioli, M., \& D'Onofrio, M. 1993, MNRAS, 265, 1013

Capaccioli, M., Caon, N., \& D’Onofrio, M. 1992, MNRAS, 259, 323

Cava, A., Bettoni, D., Poggianti, B. M., et al. 2009, A\&A, 495, 707

Cebrián, M., \& Trujillo, I. 2014, MNRAS, 444, 682

Cross, N., Driver, S., Liske, J., et al. 2004, MNRAS, 349, 576

D'Onofrio, M., Valentinuzzi, T., Fasano, G., et al. 2011, ApJ, 727, L6

D’Onofrio, M., Bettoni, D., Bindoni, D., et al. 2013a, Astron. Nachr., 334, 373

D'Onofrio, M., Fasano, G., Moretti, A., et al. 2013b, MNRAS, 435, 45

Fasano, G., \& Vio, R. 1991, MNRAS, 249, 629

Fasano, G., Amico, P., Bertola, F., Vio, R., \& Zeilinger, W. W. 1993, MNRAS, 262, 109

Fasano, G., Poggianti, B., Bettoni, D., et al. 2003, Mem. Soc. Astron. It., 74, 355

Fasano, G., Marmo, C., Varela, J., et al. 2006, A\&A, 445, 805

Fasano, G., Bettoni, D., Ascaso, B., et al. 2010, MNRAS, 404, 1490

Fasano, G., Vanzella, E., Dressler, A., et al. 2012, MNRAS, 420, 926

Franceschini, A., Silva, L., Fasano, G., et al. 1998, ApJ, 506, 600
Fritz, J., Poggianti, B. M., Cava, A., et al. 2011, A\&A, 526, A45

Fritz, J., Poggianti, B. M., Cava, A., et al. 2014, A\&A, 566, A32

Haussler, B., McIntosh, D., Barden, M., Bell, E., \& Rix, H. 2007, ApJS, 172, 633

Kormendy, J. 1977, ApJ, 218, 333

Liske, J., Lemon, D., Driver, S., Cross, N., \& Couch, W. 2003, MNRAS, 344, 307

Lotz, J. M., Madau, P., Giavalisco, M., Primack, J., \& Ferguson, H. C. 2006, ApJ, 636, 592

Metropolis, N., Rosenbluth, A. W., Rosenbluth, M. N., Teller, A. H., \& Teller, E. 1953, J. Chem. Phys., 21, 1087

Moretti, A., Poggianti, B. M., Fasano, G., et al. 2014, A\&A, 564, A138

Omizzolo, A., Fasano, G., Reverte Paya, D., et al. 2014, A\&A, 561, A111

Peng, C., Ho, L., Impey, C., \& Rix, H. 2002, AJ, 124, 266

Pignatelli, E., Fasano, G., \& Cassata, P. 2006, A\&A, 446, 373

Poggianti, B. M., Fasano, G., Bettoni, D., et al. 2009, ApJ, 697, L137

Poggianti, B. M., Calvi, R., Bindoni, D., et al. 2013a, ApJ, 762, 77

Poggianti, B. M., Calvi, R., Bindoni, D., et al. 2013b, in IAU Symp. 295, eds. D. Thomas, A. Pasquali, \& I. Ferreras, 151

Poggianti, B. M., Moretti, A., Calvi, R., et al. 2013c, ApJ, 777, 125

Saha, P., \& Williams, T. B. 1994, AJ, 107, 1295

Simard, L. 1998, in Astronomical Data Analysis Software and Systems VII, eds. R. Albrecht, R. N. Hook, \& H. A. Bushouse, ASP Conf. Ser., 145, 108

Simard, L., Trevor Mendel, J., Patton, D., Ellison, S., \& McConnachie, A. 2011, ApJS, 196, 11

Smail, I., Dressler, A., Couch, W. J., et al. 1997, ApJS, 110, 213

Valentinuzzi, T., Woods, D., Fasano, G., et al. 2009, A\&A, 501, 851

Valentinuzzi, T., Fritz, J., Poggianti, B. M., et al. 2010a, ApJ, 712, 226

Valentinuzzi, T., Poggianti, B. M., Saglia, R. P., et al. 2010b, ApJ, 721, L19

Varela, J., D’Onofrio, M., Marmo, C., et al. 2009, A\&A, 497, 667

Vulcani, B., Poggianti, B. M., Aragón-Salamanca, A., et al. 2011a, MNRAS, 412, 246

Vulcani, B., Poggianti, B. M., Dressler, A., et al. 2011b, MNRAS, 413, 921

Vulcani, B., Poggianti, B. M., Fasano, G., et al. 2012, MNRAS, 420, 1481 